\title{
CENÁRIOS QUE DESAFIAM A IMPLEMENTAÇÃO EMERGENCIAL DA POLÍTICA NACIONAL DE RESÍDUOS SÓLIDOS: UMA QUESTÃO AMBIENTAL E DE SAÚDE
}

\section{Lorena Saboya Vieira Soares* Amanda Silva Madureira**}

RESUMO: Propõe-se uma reflexão acerca dos possíveis entraves existentes na Política Nacional de Resíduos Sólidos que impedem o verdadeiro enfrentamento da sustentabilidade e a gestão adequada dos resíduos sólidos no Brasil. Os argumentos de insucesso da lei são demonstrados a partir de dados atuais que atestam a forma inadequada de disposição dos resíduos sólidos, bem como o não cumprimento das metas exigidas pela política. Sugere-se, como contribuição ao debate, algumas alternativas que, de alguma forma, propiciarão o amadurecimento da discussão, com vistas a permitir à viabilidade de implementação da política em questão.

PALAVRAS-CHAVE: Direito Ambiental; Resíduos Sólidos; Política Nacional de Resíduos Sólidos.

\section{SCENARIOS CHALLENGING THE EMERGENCY IMPLEMENTATION OF THE NATIONAL SOLID WASTE POLICY: AN ENVIRONMENTAL AND HEALTH ISSUE}

\begin{abstract}
It is proposed a reflection about the obstacles presents in the Solid Waste National Policy that prevent the sustainability confrontation to the adequate solid waste management in Brazil. The arguments of the law failure are demonstrated from current researches that attest the inadequate disposal solid waste form as well the goals required by the policy. It is suggested, as a contribution to this issue, alternatives that will allow a deepening discussion, with the objective of enabling the policy implementation.
\end{abstract}

KEYWORDS: Environmental law; Solid Waste; Solid Waste National Policy.

\footnotetext{
* Doutoranda em Políticas Públicas pela Universidade Federal do Maranhão - UFMA. Mestre em Saúde e Ambiente pela Universidade Federal do Maranhão - UFMA. Membro da Comissão de Meio Ambiente da OAB/MA. Advogada. Sócia-proprietária do escritório Correa \& Saboia Advogados. Professora de Direito Ambiental da Graduação e Pós Graduação da Universidade CEUMA. E-mail: lorenasaboia@gmail.com.

** Doutoranda em Políticas Públicas pela Universidade Federal do Maranhão - UFMA. Professora da Graduação e Pós Graduação da Universidade CEUMA.
} 


\section{INTRODUÇÃO}

A produção de materiais diversos, como fruto do desempenho das atividades humanas, sejam elas de qualquer natureza, é inerente à vida. Ao longo da história, percebe-se uma modificação na geração do que hoje se entende como resíduos sólidos, ou seja, para o atendimento das novas necessidades, ou das necessidades criadas pelo homem ${ }^{1}$, que deixaram de ser somente biológicas, os produtos precisaram ter durabilidade, e, assim, houve um aumento significativo na produção de recipientes, principalmente plásticos.

A quantidade, a sazonalidade e a heterogeneidade dos resíduos sólidos gerados ao longo do tempo podem recriar a história vivida pela humanidade. Os resíduos, em princípio exclusivamente orgânicos, foram reiteradamente substituídos por outros mais complexos, a partir do domínio da técnica e tecnologia de extração dos diferentes recursos naturais e da capacidade de beneficiamento e uso. (FIORE, 2013, p. 2)

Neste aspecto, o constante crescimento das populações urbanas, a forte industrialização, a melhoria no poder aquisitivo dos povos, de uma forma geral, vêm instrumentalizando a acelerada geração de grandes volumes de resíduos sólidos dos mais diferentes tipos.

Ferreira (2005) descreve que a nossa civilização chega ao limiar do século XXI como a civilização dos resíduos, marcada pelo desperdício e pelas contradições de um desenvolvimento industrial e tecnológico sem precedentes na história da humanidade.

Daniel Véras Ribeiro e Márcio Raymundo Morelli registram que estas grandes quantidades de resíduos gerados, bem como leis que não punem exemplarmente os geradores que não os gerenciam eficazmente, constituem-se num dos grandes problemas da sociedade moderna. (RIBEIRO e MORELLI, 2009, p.3).

Esse cenário acaba sendo comum em todas as partes do mundo, especialmente porque as atividades humanas, em era globalizada, acabam sendo comuns, com os mesmos resultados (ARENDT, 2009).

Refletir este contexto é de suma importância quando se depara com uma sociedade mundializada (CHESNAIS, 1995), em que as formas de relação entre os homens vão ganhando

\footnotetext{
${ }^{1}$ Agnes Heller, 1978
} 
novos contornos, os quais podem ter diferentes consequências dependendo da localidade ou cultura.

No entanto, no que se refere à geração de resíduos, o que se percebe é uma realidade comum, posto que, em todas as partes do mundo, pessoas então consumindo, cada vez mais, e gerando resíduos mais difíceis de serem tratados.

Parece uma conta irracional, na medida em que o que se consome retorna em forma de resíduo, num desafio muito maior do que a própria produção.

Segundo Annes (2012), os resíduos são considerados como um sinal de produção ineficiente. Já, para a Associação Brasileira de Normas Técnicas - ABNT, resíduos são os restos das atividades humanas, considerados pelos geradores como inúteis, indesejáveis ou descartáveis, podendo apresentar-se no estado sólido, semi-sólido ou líquido, desde que não seja passível de tratamento convencional (ABNT, 2004).

A geração de resíduos passou a representar um dos resultados negativos do processo de produção em larga escala, consequência do sistema capitalista, tendo como palco de evidência o ambiente urbano ${ }^{2}$, ou seja, é nas cidades que a aparência explícita da forma de tratamento dos resíduos sólidos fica latente.

A produção de resíduos, assim, é condicionada às necessidades da população, que, neste aspecto, é quem dá o tom ao quantitativo gerado e às drásticas consequências relacionadas, ou seja, o aumento da produção de resíduos acaba acompanhando o crescimento populacional.

No Brasil, segundo a Associação Brasileira de Empresas de Limpeza Pública e Resíduos Especiais (Abrelpe, 2016) ${ }^{3}$, nos últimos 10 anos, a população aumentou 9,65\% e o volume de resíduos $21 \%$.

Apesar do expressivo crescimento do número de resíduos, que pode ser atribuído, inclusive, à modificação social e ascensão da população para a classe média, consequência do aquecimento da economia, $38 \%$ dos brasileiros (78 milhões de pessoas) continuam sem acesso a serviços de tratamento e destinação adequada de resíduos (ABRELPE, 2016).

\footnotetext{
${ }^{2}$ Segundo as Nações Unidas, o número de pessoas vivendo em cidades deve crescer de 3,5 bilhões em 2012 para 6,3 bilhões até 2050. Disponível em <www.onu.org.br>. MAR 2018.

${ }^{3}$ Disponível em http://www.abrelpe.org.br/panorama_apresentacao.cfm. MAR 2018.
} 
A Organização das Nações Unidas (ONU, 2015) ${ }^{4}$ contabilizou a existência de 1.775 lixões no Brasil, e, muitos deles, ainda com pessoas catando materiais em condições insalubres e degradantes à dignidade humana 5 .

O que não é diferente da realidade mundial, onde somente metade da população mundial é atendida por coleta de resíduos sólidos (ISWA, 2013) ${ }^{6}$.

As previsões de futuro não são nem um pouco animadoras, visto que, segundo o Programa das Nações Unidas para o Meio Ambiente - PNUMA ${ }^{7}$, para o ano de 2025, esperase um aumento na produção de resíduos sólidos, no mundo, o que inclui o Brasil, de 1,3 bilhão toneladas/ano para 2,2 bilhões toneladas/ano, sendo que quase a metade desse total é gerada por menos de 30 países, os mais desenvolvidos.

Frise-se que a geração de resíduos, ao longo do tempo, torna-se diferente no que tange à tipologia. Com o avanço da tecnologia, há a consequente produção de resíduos cada vez mais difíceis de ser tratados, ou descartados na natureza, sem ocasionar impactos ambientais significativos, como nos recursos hídricos, no solo e nas cidades.

O tão necessário enfrentamento legislativo da problemática dos resíduos sólidos, no Brasil, ocorreu, de fato, apenas em 2010, com a publicação da Lei 12.305, que instituiu a Política Nacional de Resíduos Sólidos, apesar das primeiras iniciativas legislativas para a definição de diretrizes à área de resíduos sólidos surgirem no final da década de 80 (Projeto de Lei do Senado no 354, de 1989). Desde então, foram elaborados mais de 70 Projetos de Lei.

Segundo Peixoto (2010), somente em 2010, após mais de duas décadas de discussões, prazo excessivamente amplo, inúmeras comissões e trâmites diversos, para um país que descarta cerca de 150 mil toneladas de lixo por dia, foi aprovada a Lei da Política Nacional de Resíduos Sólidos - PNRS.

A PNRS pretendeu reduzir a geração de resíduos, incentivar a reciclagem e determinar o que fazer com o lixo remanescente. Buscou, também, obrigar fabricantes, importadores, distribuidores e vendedores a recolher embalagens usadas.

A "nova" ${ }^{8}$ lei fundamenta-se no princípio de responsabilidade compartilhada", ao

\footnotetext{
${ }^{4}$ Disponível em http://www.grida.no/publications/166.htm. FEV 2018. reclusão de 1 a 5 anos.

${ }^{6}$ Disponível em <http://www.iswa.org >. MAR 2018.

${ }^{7}$ Disponível em <http://www.pnuma.org.br>. MAR 2018.

8 Já com 7 anos de existência.

${ }^{9}$ Art. $3^{\circ}$ - inciso XVII da Lei $12.305 / 2010$.
}

${ }^{5} \mathrm{O}$ artigo 47 da Lei 12.305 de 02 de agosto de 2010, que institui a Política Nacional de Resíduos Sólidos, proíbe a destinação ou disposição final de resíduos sólidos ou rejeitos in natura a céu aberto, e o artigo 54, V, da Lei de Crimes Ambientais, $n^{\circ}$ 9.605, de 12 de fevereiro de 1998, considera esta conduta criminosa, punida com pena de 
propiciar oportunidades de cooperação entre os entes da Federação, o setor produtivo e a sociedade em geral.

A PNRS estabeleceu, ou pelo menos se propôs a estabelecer, novos rumos para a gestão e o gerenciamento dos resíduos sólidos no país, de forma integrada e compartilhada. No entanto, os desafios que se apresentam são de grande ordem, já que as fragilidades da Lei, durante esses anos de vigência, estão cada vez mais latentes, especialmente quando se avalia os resultados e metas inalcançáveis.

O estudo propõe, assim, uma reflexão sobre os desafios de implementação Política Nacional de Resíduos Sólidos que impedem o verdadeiro enfrentamento da sustentabilidade e a gestão adequada dos resíduos sólidos no Brasil e sugere, como contribuição ao debate, algumas alternativas que propiciarão o amadurecimento da discussão, com vistas a permitir à viabilidade de implementação da política em questão.

\section{O OBJETIVO DO CONSUMO SUSTENTÁVEL PREVISTO NA AGENDA 21 E SUA INFLUÊNCIA NA PNRS}

O consumo sustentável tornou-se um objetivo a ser alcançado, desde a Conferência de Estocolmo de 1972, quando, pela primeira vez, o assunto foi tratado com a devida importância. Desde então a limitação natural dos recursos do meio ambiente em face do progresso mundial tornaram-se indispensáveis.

Em 1992 foi realizado no Rio de Janeiro, Brasil a Conferência das Nações Unidas sobre o Meio Ambiente e o Desenvolvimento, onde foi assinado por 179 países a Agenda 21 Global, termo cunhado para representar o desejo de mudança a ser alcançado até o século $\mathrm{XXI}^{10}$.

Em seu capítulo quarto, itens 4.18 e 4.19, , a Agenda trata da mudança dos padrões de consumo, como medida a viabilizar a redução de resíduos em consonância com o desenvolvimento:

4.18. A redução do volume de energia e dos materiais utilizados por unidade na produção de bens e serviços pode contribuir simultaneamente para a mitigação da pressão ambiental e o aumento da produtividade e competitividade econômica e industrial. Em decorrência, os Governos, em cooperação com a indústria, devem

\footnotetext{
10 BRASIL. Ministério do Meio Ambiente. Agenda 21 Global. Disponível em: http://www.mma.gov.br/responsabilidade-socioambiental/agenda-21/agenda-21-global.
} 
intensificar os esforços para utilizar a energia e os recursos de modo economicamente eficaz e ambientalmente saudável, como se segue:

$[\ldots]$

(f) Redução ao mínimo da geração de resíduos

4.19. Ao mesmo tempo, a sociedade precisa desenvolver formas eficazes de lidar com o problema da eliminação de um volume cada vez maior de resíduos. Os Governos, juntamente com a indústria, as famílias e o público em geral, devem envidar um esforço conjunto para reduzir a geração de resíduos e de produtos descartados, das seguintes maneiras:

(a) Por meio do estímulo à reciclagem no nível dos processos industriais e do produto consumido;

(b) Por meio da redução do desperdício na embalagem dos produtos;

(c) Por meio do estímulo à introdução de novos produtos ambientalmente saudáveis;

(d) Auxílio a indivíduos e famílias na tomada de decisões ambientalmente saudáveis de compra.

Com base nos objetivos constituídos na Agenda 21 Global de 1992, o Brasil, em 2002, estabeleceu seu próprio plano de sustentabilidade, a Agenda 21 Brasileira, com participação da população. O plano pátrio dispõe de 21 metas prioritárias afim de alcançar o desenvolvimento sustentável.

O Objetivo 1 é a Produção e Consumo Sustentáveis contra a Cultura do Desperdício e tem o propósito de reduzir o as perdas produtivas promovendo uma política de conscientização social da população através da mudança de comportamento.

Este objetivo foi, sem dúvida alguma, um dos maiores influenciadores na elaboração da PNRS, já que a Lei prevê a prevenção e a redução na geração de resíduos, tendo como proposta a prática de hábitos de consumo sustentável e um conjunto de instrumentos para propiciar o aumento da reciclagem e da reutilização dos resíduos sólidos e a destinação ambientalmente adequada dos rejeitos ${ }^{11}$.

É notório que a questão da sustentabilidade está diretamente relacionada a redução do desperdício e consequentemente a diminuição na produção de resíduos. Logo, percebe-se esta relação intrínseca presente na Lei 12.305/2010, a qual traz, expressamente, em seu artigo $3^{\circ}$, inciso XIII ${ }^{12}$, o conceito de padrões sustentáveis de produção e consumo.

Em consonância com as modernas práticas sustentáveis, o legislador buscou estabelecer, enquanto resíduo, aquilo que, de alguma forma, poderia ser reaproveitado, seja pela reciclagem ou compostagem.

\footnotetext{
${ }^{11}$ BRASIL, Ministério do Meio Ambiente.

${ }^{12}$ Art. $3^{\circ}$. Para os efeitos desta Lei, entende-se por: XIII - padrões sustentáveis de produção e consumo: produção e consumo de bens e serviços de forma a atender as necessidades das atuais gerações e permitir melhores condições de vida, sem comprometer a qualidade ambiental e o atendimento das necessidades das gerações futuras.
} 
Édis Milaré destaca (2015, p. 1187) que tudo o que é descartado em decorrência das atividades sociais humanas é considerado resíduo sólido. A lei não enfrenta se o descarte é promovido em função do esgotamento das características e das propriedades originais da matéria, substância, objeto ou bem, ou se o descarte é promovido em função de uma análise subjetiva do indivíduo daquilo que pode ser considerado inservível para a sociedade humana.

Na Agenda 21 global, há, também, em seu vigésimo primeiro capítulo, as diretrizes para a gestão dos resíduos sólidos de forma compatível com a preservação ambiental:

O manejo ambientalmente saudável desses resíduos deve ir além do simples depósito
ou aproveitamento por métodos seguros dos resíduos gerados e buscar resolver a
causa fundamental do problema, procurando mudar os padrões não sustentáveis de
produção e consumo. Isso implica na utilização do conceito de manejo integrado do
ciclo vital, o qual apresenta oportunidade única de conciliar o desenvolvimento com
a proteção do meio ambiente.

Para atingir tal objetivo, um planejamento dos resíduos sólidos precisa incorporar então: (a) Redução ao mínimo dos resíduos; (b) Aumento ao máximo da reutilização e reciclagem ambientalmente saudáveis dos resíduos; (c) Promoção do depósito e tratamento ambientalmente saudável dos resíduos; (d) Ampliação do alcance dos serviços que se ocupam dos resíduos. (LOPES, 2006, p. 52).

Para o fiel entendimento e execução favorável, a Lei baseou-se, também, em diversos outros princípios presentes, direta ou indiretamente, na Agenda 21, como: o Desenvolvimento Sustentável, considerado um de seus pilares, conceituado pela Comissão Mundial sobre o Meio Ambiente e Desenvolvimento como "um desenvolvimento que faz face às necessidades das gerações presentes sem comprometer a capacidade das gerações futuras na satisfação de suas próprias necessidades"; a Cooperação entre as diferentes Esferas do Poder Público, o Setor Empresarial e demais segmentos da Sociedade, permitindo-se o estreitamento dos laços entre o poder público, empresários e a sociedade em geral, afim de aproximar o diálogo entre os setores e o princípio da Responsabilidade Compartilhada pelo Ciclo de Vida dos Produtos, que estabelece uma forma de elasticidade do alcance de atores e condutas que possam resultar em danos ao meio ambiente, ou seja, de toda cadeia produtiva.

Percebe-se, assim, a influência clara da Agenda 21 como base principiológica e conceitual, bem como para as metas definidas na PNRS, tendo o consumo sustentável como pilar.

\section{OS DESAFIOS DA PRNS NA IMPLEMENTAÇÃO DA GESTÃO INTEGRADA DOS RESÍDUOS SÓLIDOS}

Rev. de Direito e Sustentabilidade | e-ISSN: 2525-9687 | Porto Alegre | v. 4 | n. 2 | p. 74 - 89 | Jul/Dez. 2018 
Como forma de cumprimento dos objetivos estabelecidos na PNRS, o legislador fornece importantes instrumentos de concretização de metas, como a gestão integrada dos resíduos sólidos.

O conceito de gestão está relacionado a uma amplitude de ação e tomada de decisão. A partir da visão holística dos cenários construídos, criam-se posicionamentos ou tomadas de decisão pelo administrador, que irão orientá-lo na elaboração de um planejamento estratégico para se chegar a um determinado objetivo. (LIMA, 2001).

No que se refere aos resíduos sólidos, o objetivo principal da aplicação da gestão é alcançar, dentro de parâmetros definidos, a redução da geração dos resíduos, o seu correto tratamento, bem como a destinação final adequada.

Sendo assim, a gestão integrada dos resíduos sólidos percebe os resíduos não só no momento do seu descarte, mas sim, desde a primeira tomada de decisão, que é o consumo.

Por esta razão, o objetivo da Agenda 21, acima discutido, influencia todo aspecto da Lei, especialmente no que tange ao olhar integrado da geração dos resíduos e da sua impossibilidade de fragmentação.

O termo "integrado", tanto no âmbito da Gestão como no Gerenciamento dos Resíduos Sólidos, está relacionado ao conjunto de ações, sejam elas operacionais, financeiras e de planejamento, que sejam inter-relacionadas e compatíveis com a realidade local. Para a gestão dos resíduos sólidos, é mais aceita e praticada por se tratar de um sistema que seleciona as técnicas e tecnologias apropriadas (ZIA; DEVADAS, 2008).

Segundo o Compromisso Empresarial para Reciclagem ${ }^{13}$, o termo Gerenciamento Integrado de Resíduos Sólidos contempla a parte técnica dos processos, ou seja, o conjunto articulado de ações normativas, operacionais, financeiras e de planejamento que uma administração municipal desenvolve para coletar, transportar e dispor os resíduos gerados na cidade.

Percebe-se que a gestão integrada dos resíduos sólidos permite a interação de diversas ações e atores, o que cristaliza a presença da sustentabilidade em todo seu contexto.

A ferramenta da gestão integrada possibilita que os gestores públicos possam efetuar o controle total da produção, impondo responsabilidades individualizadas a cada participante (responsabilidade compartilhada).

\footnotetext{
${ }^{13}$ CEMPRE, 2015
} 
O princípio da responsabilidade compartilhada estabelecido, apesar de envolver diversos atores participantes, impõe aos gestores municipais a maior carga de cumprimento, ou seja, compete aos Municípios a implementação da gestão integrada e o cumprimento das principais metas estabelecidas pela PNRS.

Uma das metas priorizadas pela Lei foi o encerramento de lixões, previsto, inicialmente, para 2 de agosto de 2014, e, diante do quase total descumprimento, foi prorrogado para 20121.

Além da erradicação dos lixões, outro objetivo incumbido aos Municípios, foi a elaboração de seus planos municipais de gestão integrada de resíduos sólidos, conforme o art. 55 da Lei, implicando, inclusive, em sérias consequências em caso de descumprimento.

A partir de 2 de agosto de 2012, os estados e municípios que não tiverem seus planos elaborados não poderão ter acesso a recursos da União, ou por ela controlados, para serem utilizados em empreendimentos e serviços relacionados à gestão de resíduos sólidos. (MMA, 2016).

O Município de São Luís, por exemplo, até os dias atuais, não elaborou o seu Plano, o que impede, em tese, que este Município possa pleitear parcerias financeiras com a União para investimentos em gestão integrada de resíduos.

Desta maneira, questiona-se as razões pelas quais muitos Planos ainda não foram entregues pelo Municípios Brasileiros.

De acordo com a Pesquisa de Informações Básicas Municipais - MUNIC, ano base 2013 (IBGE, 2014), apenas 1.865 municípios declararam possuir planos de gestão integrada de resíduos sólidos nos termos da PNRS, de um total de 5.561 Municípios.

Em 7 anos de existência da Lei, não se atingiu nem 50\% do cumprimento da meta de elaboração dos Planos Municipais, que impedem a execução da gestão integrada dos resíduos sólidos no ambiente local.

\section{PNRS E AS POUCAS METAS IMPLEMENTADAS: FRACASSO OU INCORRETA APLICAÇÃO DA GESTÃO INTEGRADA?}

Acompanhar a implementação de metas em quaisquer que sejam as áreas nas políticas públicas não é tarefa fácil. Isto decorre, em certa medida, do grau de incerteza em que a política é construída. Toma-se como exemplo, o conceito depreendido de Dye (DYE, 2015) no que se refere ao campo das políticas públicas. Para Dye, a política pública é aquilo que governos fazem 
ou deixam de fazer (DYE, 2015).

O modo de resolver os problemas irá depender da capacidade político-administrativa do Estado de conciliar interesses e divergências entre os seus membros. Assim, vislumbra-se a política pública como uma "diretriz para enfrentar um problema público" (SECCHI, 2013, p. 2).

Embora não exista uma definição perfeita da política pública, é possível chegar a um conjunto de elementos norteadores, tais como a centralidade do governo, o papel das instituições, a presença de grupos sociais, interesses e as interações necessárias que envolvem a natureza da política pública e seus processos (SOUZA, 2006). Fruto do pós-Guerra Fria, a política pública progressivamente vai proporcionar a viabilidade de análise técnica das decisões de governo por parte de pesquisadores e formulações científicas (SOUZA, 2006).

Porém, nesse ambiente dinâmico e contraditório, os problemas que atingem os membros de uma sociedade precisam integrar a agenda pública e, por consequência, ter o reconhecimento da sociedade vislumbrando as possibilidades de ação por parte do governo sujeito aos grupos de pressão, aos partidos políticos, mídia, opinião pública para que revista-se de um objeto de ação política.

Como fazer isso? De que maneira os problemas são levados à mesa de negociação e, ao fim de todo o processo em torno das possíveis soluções, a sociedade pode perceber que suas necessidades são satisfeitas ou ao menos, minoradas? Ou ainda: por que alguns problemas aparecem na agenda governamental como objetos de ação e outros não?

Esses questionamentos tem acompanhado o processo de compreensão das políticas públicas não como um conjunto harmônico, mas circunscrito a lógicas diferenciadas de poder que pode transpor determinado objeto de ação política a práticas residuais e, portanto, sem mudança na realidade.

Por essa razão, toda e qualquer política pública se operacionaliza em planos, metas, resoluções que devem, por sua vez, estabelecer uma série de metas a serem alcançadas ao longo do tempo. No país, por exemplo, acompanhar a implementação de metas reveste-se de uma tarefa hercúlea uma vez que o Brasil não possui certa tradição na avaliação de seus programas de forma periódica.

Nesse sentido, discorrer sobre as metas relacionadas à PNRS só faz sentido se houver a compreensão de todos os sujeitos envolvidos no processo de gestão de resíduos sólidos. Talvez, a magnitude geográfica do país cause uma certa dificuldade em operacionalizar a gestão de resíduos devido à fragmentariedade da rede envolvida. Por exemplo, diante da diversidade de 
produção de resíduo no país, como garantir aos Municípios a operacionalização da PNRS?

A PNRS trouxe, além de uma base fundamental para a gestão de resíduos sólidos no Brasil, uma série de prazos e metas ousadas, tanto para o Poder Público, como para os geradores de resíduos responsáveis pela sua adequada destinação.

Os desafios impostos foram estabelecidos com base na possibilidade de aplicação da gestão integrada dos resíduos sólidos, com participação efetiva de todos os atores envolvidos, com o objetivo de promover o controle desde a geração até o adequado descarte.

Neste contexto, importante refletir a aplicabilidade da Lei na perspectiva da sustentabilidade a que ela se propôs, com vistas a identificar os reais problemas que impedem a fiel execução do gerenciamento integrado.

Ao fazer-se uma primeira reflexão acerca da Lei e sua implementação, percebe-se que poucos avanços foram dados.

Segundo dados apresentados pela Associação Brasileira de Empresas de Limpeza Pública e Resíduos Especiais, em 2013, 41,74\% dos resíduos sólidos tem destinação inadequada $^{14}$.

No caso específico do Estado do Maranhão, existe, ainda, segundo a mesma pesquisa, a exclusão de $33.9 \%$ da população da coleta de resíduos sólidos, ou seja, apenas um universo de $66 \%$ da população goza do serviço, cabendo aos demais a utilização de técnicas insalubres e inadequadas, como a queima a céu aberto dos resíduos sólidos.

Esta realidade não é diferente das outras regiões do Brasil, que enfrentam os mesmos problemas relacionados a implementação da Lei. Estimativas do Movimento Nacional dos Catadores de Materiais Recicláveis (MNCR, 2012) apontam que existam cerca de 800 mil catadores em atividade no Brasil, a maior parte trabalhando nas ruas e nos lixões ${ }^{15}$.

Ressalta-se que os principais impactos de práticos de cumprimento da PNRS recaem sobre os Municípios da Federação, os quais são detentores, segundo a Constituição Federal de $1988^{16}$, da competência para a gestão da limpeza pública urbana e do consequente controle dos resíduos em seus territórios.

\footnotetext{
${ }^{14}$ Dados obtidos em <http://www.abrelpe.org.br/_download/informativo_recuperacao_energetica.pdf $>$. MAR 2017.

${ }^{15}$ Disponível em <http://www.mncr.org.br/mncr/artigos?b_start:int=30>. FEV 2017.

${ }^{16}$ Artigo 30 da CF.
} 
Ademais, a promoção da adequada gestão de resíduos envolve, o ordenamento territorial, mediante planejamento e controle do uso, do parcelamento e da ocupação do solo urbano, de igual competência Municipal.

Segundo dados do IPEA (2012), considerando-se uma amostra de 256 Municípios, a despesa com manejo de Resíduos Sólidos Urbanos corresponde, em média, a 5,3\% das despesas correntes das prefeituras.

Significa que, diante de uma média de despesa com folha de pagamento correspondente a $50 \%$ dos orçamentos municipais ${ }^{17}$, o impacto atual dos custos com coleta e tratamento de resíduos, sem levar em consideração as metas da PNRS, como construção de aterros e elaboração de planos, já é extremamente considerável, pois existem outras demandas prioritárias e importantes como educação e saúde.

Portanto, o que se constata é que, se as principais metas estipuladas na PNRS recaem sobre os Municípios, e estes já possuem um déficit de orçamento para a gestão que hoje já realizam, é praticamente impossível atender ao exigido com as ferramentas atuais existentes. Assim, a preocupação com o cenário aumenta, a cada dia que passa, e, quanto mais demoradas as soluções, mais onerosas.

Importante trazer à baila o papel, neste contexto, da gestão integrada intermunicipal dos resíduos sólidos, através dos consórcios públicos, como uma relevante ferramenta.

Muitos municípios brasileiros encontram dificuldades que são quase insolúveis quando enfrentadas isoladamente para planejar, regular e promover a adequada operação dos serviços de manejo de resíduos sólidos. É, sobretudo, em razão da necessidade de superar essas deficiências estruturais que se torna necessário considerar a gestão regionalizada por meio dos consórcios públicos, buscando, assim, a sustentabilidade dos investimentos. (MAINELLO, BRITTO e VALLE, 2018).

Segundo a Abrelpe (2015), os custos de operação e manutenção para metas definidas no Plano Nacional de Resíduos Sólidos chega, em media, a R \$ 15,59 bilhões. Para universalizar a destinação final adequada dos resíduos sólidos, o Brasil precisa investir R \$ 11,6 bilhões até 2031.

Se o cenário aponta a necessidade de altos investimentos para que se alcance um cumprimento satisfatório de metas, talvez, a gestão integrada intermunicipal seja, de fato, uma estratégia extremamente possível.

\footnotetext{
${ }^{17}$ Conforme o que estabelece a Lei de Responsabilidade Fiscal.
} 
O modelo cooperativo traz uma série de vantagens aos municípios na gestão de RSU, como o maior controle sobre o tratamento e a disposição final; o fortalecimento dos potenciais de reuso, acrescendo a capacidade de coleta, triagem e então reciclagem dos materiais; o incentivo à organização de catadores em cooperativas com o consequente melhoramento das condições de trabalho e de vida (MAINELLO, BRITTO e VALLE, 2018 apud MORAES, 2012)

A gestão integrada dos resíduos permite o alcance de resultados satisfatórios quando corretamente implementada. Neste contexto, importante refletir acerca dos aspectos principais que impedem o avanço das metas estabelecidas na Lei e as alternativas possíveis que viabilizem a sua implementação, de forma a garantir o meio ambiente saudável, para as presentes e futuras gerações.

\section{CONSIDERAÇÕES FINAIS}

A presente reflexão apresentou a realidade que o Brasil vive hoje no contexto dos resíduos sólidos e a dificuldade encontrada na aplicabilidade na PNRS, demonstrando-se que a legislação, isoladamente, não foi suficiente para a mudança no quadro geral da situação inadequada dos resíduos no Brasil.

Não restam dúvidas que o descarte de resíduos sólidos no meio ambiente gera poluição e impactos de toda ordem, principalmente nas áreas urbanas de baixa renda, as quais, além de serem desprovidas da coleta de resíduos, convivem com a contaminação de solos e corpos d'água, bem como da produção de vegetais e animais que nestes ambiente convivem.

Nos municípios em que a pobreza é endêmica, o analfabetismo dominante, o saneamento básico não existe, a condições da saúde públicas são lastimáveis e a consciência política e ambiental não se conhecem, será um tremendo desafio (GODOY, 2013).

Os dados alarmantes atestam, de forma clara, que a velocidade de geração de resíduos não acompanha a correspondente evolução de tecnologias ou políticas de redução do consumo e destinação ambientalmente adequada. É a prova pura de uma "não política".

O desafio que se apresenta em torno da temática, é, justamente, a complexidade em que os resíduos sólidos estão imersos, não sendo algo de fácil resolução, incluindo, até mesmo, os direitos fundamentais da pessoa humana relacionados ao seu bem-estar e a uma vida digna, como o direito ao meio ambiente ecologicamente equilibrado, à saúde, à habitação, ao lazer, à segurança, ao trabalho e às cidades sustentáveis. (OKADA, 2009). 
A falta de promoção e execução de políticas públicas sérias, bem como não aplicação adequada da gestão integrada dos resíduos sólidos, são os principais fatores que concorrem para o cenário.

A participação social nesta problemática dos resíduos deve ser muito mais ativa do que a vista até agora, já que, sem a devida contribuição do cidadão, não há como comemorar avanços.

Outro aspecto, importante, também, seria a tentativa de encontrar saídas de gestão que otimizem custos, viabilizem tecnologias inovadoras e eficientes, promovam parcerias duradouras que, de fato, viabilizem a integralidade das políticas de resíduos no Municípios, especialmente naqueles que dispõem de menos recursos, com sustentabilidade e adequação para o futuro.

É notório que a legislação brasileira sobre os resíduos sólidos já está em uma fase avançada de consolidação, com diretrizes, princípios, objetivos e instrumentos bem definidos, no entanto, a aproximação com a realidade ainda deixa muito a desejar.

Restou claro, portanto, que o controle dos resíduos sólidos, depende, prioritariamente, da elaboração e implementação de políticas públicas, pautadas em pesquisas que garantam a eficácia dos programas desempenhados, através da análise de resultados e da concepção/desenho da própria política.

É inadmissível continuar esperando o futuro que vier, sem planejamento ou gestão responsável.

\section{REFRÊNCIAS BIBLIOGRÁFICAS}

ABNT, Associação Brasileira de Normas e Técnicas. NBR 10.004. Resíduos sólidos: classificação. Rio de Janeiro: ABNT, 2004.

ABRELPE, Associação Brasileira de empresas de limpeza pública e resíduos especiais. Panorama nos resíduos sólidos no Brasil 2015. Disponível em <http://www.abrelpe.org.br/panorama_apresentacao.cfm.htm>.

ALMEIDA, Lia de Azevedo. A formulação da política nacional de resíduos sólidos: uma análise orientada pela complexidade. Tese (Doutorado em Administração). Universidade de Brasília - UnB. Brasília, 2016.

ANNES, J. B. Contabilidade ambiental na indústria- uma ferramenta para a produção mais limpa/Sistema de Gestão Ambiental. Disponível em: 〈http://www. stoa.usp.br〉. 
ARENDT, Hannah. A condição humana. Rio de Janeiro: Forense Universitária, 2009.

BRASIL, Lei $\mathrm{n}^{\circ} 12.305$ de 02 de agosto de 2010. Política Nacional de Resíduos Sólidos. Disponível em <http://www.planalto.gov.br/ccivil_03/_ato2007-2010/2010/lei/112305.htm>.

BRASIL. Ministério do Meio Ambiente. Agenda 21 Global. Disponível em: <http://www.mma.gov.br/responsabilidade-socioambiental/agenda-21/agenda-21global.htm>.

CEMPRE, Compromisso Empresaria para Reciclagem. Cempre Review 2015. Disponível em $<$ http://cempre.org.br/artigo-publicacao/artigos.htm>.

FERREIRA, J. A. Resíduos sólidos e lixo hospitalar: Uma discussão ética. Cad.Saúde Públ. v.11 n.2, Rio de Janeiro, abr./jun. 2005.

DYE, Thomas. Understanding public policy. 13 ${ }^{\mathrm{a}}$ ed. Boston: Pearson, 2011, cap. 1 Policy analysis: what governments do, why they do it, and what diffence it makes. p. $1-10$.

FIORE, Fabiana Alves. A gestão municipal de resíduos sólidos por meio de redes técnicas. Campinas, SP. Tese (Doutorado) - Universidade Estadual de Campinas - Faculdade de Engenharia Civil, Arquitetura e Urbanismo. Disponível em <http://repositorio.unicamp.br/bitstream/REPOSIP/257718/1/Fiore,\%20Fabiana\%20Alves_D. pdf.htm>.

GODOY, Manuel Rolando Berríos. Dificuldades para aplicar a Lei da Política Nacional de Resíduos Sólidos no Brasil. Caderno de Geografia. Universidade Estadual Paulista, 2013.

HELLER, Agner. Teoría de las necesidades en Marx. Traducido por J. F. Yvars Ediciones Península, Barcelona, 1978 Segunda edición, 1986

IBGE, Instituto Brasileiro de Geografia e Estatística. Pesquisa de Informações Básicas $\begin{array}{llll}\text { Municipais } & \text { - } & 2013 .\end{array}$ <http://www.ibge.gov.br/home/estatistica/economia/perfilmunic/.htm>.

IPEA, Instituto de Pesquisa Econômica Aplicada. Diagnóstico dos Resíduos Sólidos Urbanos. $2012 . \quad$ Disponível em <http://www.ipea.gov.br/agencia/images/stories/PDFs/relatoriopesquisa/121009_relatorio_res iduos_solidos_urbanos.pdf.htm>.

LIMA, J. D. Gestão de resíduos sólidos urbanos no Brasil. Campina Grande: ABES, 2001.

LOPES, Luciana. Gestão e Gerenciamento Integrados dos Resíduos Sólidos Urbanos: alternativas para pequenos municípios. São Paulo, SP. Dissertação (mestrado). Programa de Pós Graduação em Geografia Humana. Universidade de São Paulo. 2006.

MAINELLO, Antonella; BRITTO, Ana Lucia Nogueira de Paiva e VALLE, Tatiana Freitas. Implementação da Política Nacional de Resíduos Sólidos. Revista de Administração Pública: Rio de Janeiro 52 (1): 24-51, jan-fev, 2018.

MNCR, Movimento Nacional dos Catadores de Materiais Recicláveis. Plataforma em Defesa da Coleta Seletiva. 2012. Disponível em <http://www.mncr.org.br/artigos/lataforma-em- 
defesa-da-coleta-seletiva.htm>.

MILARÉ, Édis. Direito do Ambiente. 10 ed. Editora Revista dos Tribunais, 2015.

NALINI, José Renato. Ética Ambiental. 4 ed. Editora Revista dos Tribunais, 2015. 304 p.

OKADA, Denise Setsuko et al. Resíduos - Consumo e Pós Consumo. Cidades Sustentáveis no Brasil e sua Tutela Jurídica. Rio de Janeiro: Lumem Juris, 2009.

ONU, Organização das Nações Unidas. Waste Crime - Waste Risks: Gaps in Meeting the Global Waste Challenge. Disponível em <http://www.grida.no/publications/166.htm>.

PEIXOTO, Cássio dos Santos. Política Nacional de Resíduos Sólidos (PNRS) e adequação das Empresas. Disponível em: $<$ http://noticias.ambientebrasil.com.br/artigos/2010/07/30/58284-politica-nacional-deresiduos-solidos-pnrs-e-adequacao-das-empresas.html> Acesso em: 14/10/2010.

PNUMA, Programa das Nações Unidas para o Meio Ambiente. Substâncias nocivas e Resíduos. Disponível em <http://web.unep.org/substâncias-nocivas-eres\%C3\%ADduos.htm>.

RIBEIRO, Daniel Véras e MORELLI, Márcio Raymundo. Resíduos sólidos: problema ou oportunidade? Rio de Janeiro: Interciência, 2009.

SECCHI, Leonardo. Políticas Públicas: conceitos, esquemas de análise, casos práticos. $2^{\mathrm{a}}$ ed. São Paulo: Cengage Learning, 2013, p. 1 - 22.

SOUZA, Lincoln Moraes. A agenda e as agendas no Brasil. Cronos, Natal - RN, v.7, n. 1, jan/jun. 2006, p. $79-101$.

ZIA, H.; DEVADAS, H. Urban solid waste management in Kanpur: Opportunities and perspectives. Habita Internacional, 2008. 